\title{
Boundary Integral Formulation of Electric Fields in Level Set Simulations of Charged Droplets
}

\author{
Anton VanderWyst* \\ Department of Aerospace Engineering, University of Michigan, Ann Arbor, MI, 48109 \\ Andrew Christlieb ${ }^{\dagger}$ \\ Department of Mathematics, University of Michigan, Ann Arbor, MI, 48109 \\ Mark Sussman \\ Department of Mathematics, Florida State University, Tallahassee, FL 32306-4510 \\ Iain D. Boyd ${ }^{\S}$ \\ Department of Aerospace Engineering, Univeristy of Michigan, Ann Arbor, MI 48109
}

\begin{abstract}
Field emission electric propulsion (FEEP) thrusters are useful as $\mu \mathrm{N}, \mu$-rad attitude control devices for satellites due to their very high potential specific impulse. FEEPs generate thrust by application of a strong electric field to pull liquid propellant such as indium off a tungsten needle. However, this thrust can vary significantly depending on whether ions or droplets are emitted from the needle tip. In this study, we simulate in 2D the emission of charged indium droplets from the tip. The boundary integral method (BIM) is used to rapidly and accurately calculate the electric field on the fluid surface, which is then advected forward in time using level sets. The effects of surface tension, viscosity, electrode location and electric field on the evolution of a droplet are analyzed.
\end{abstract}

\section{Nomenclature}

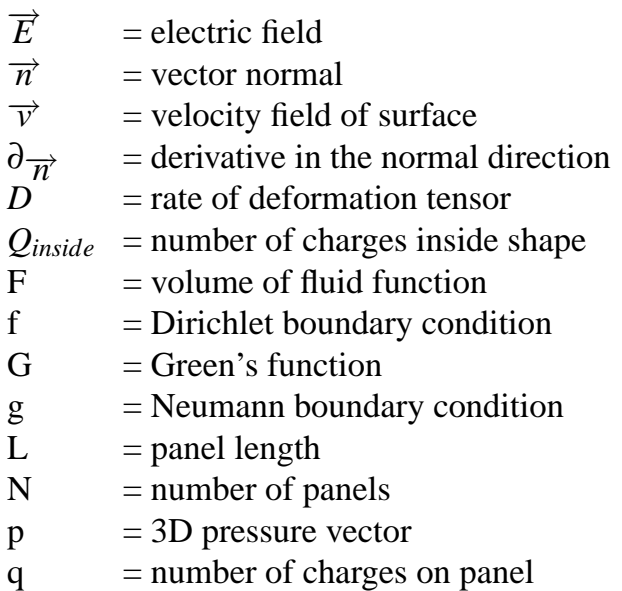

\section{Subscripts}

$=$ time change of vector

${ }^{*}$ Graduate Research Assistant. Student Member AIAA. Email: antonv@umich.edu

${ }^{\dagger}$ Assistant Professor. Member AIAA

${ }^{\ddagger}$ Assistant Professor

$\S$ Professor. Associate Fellow AIAA. 


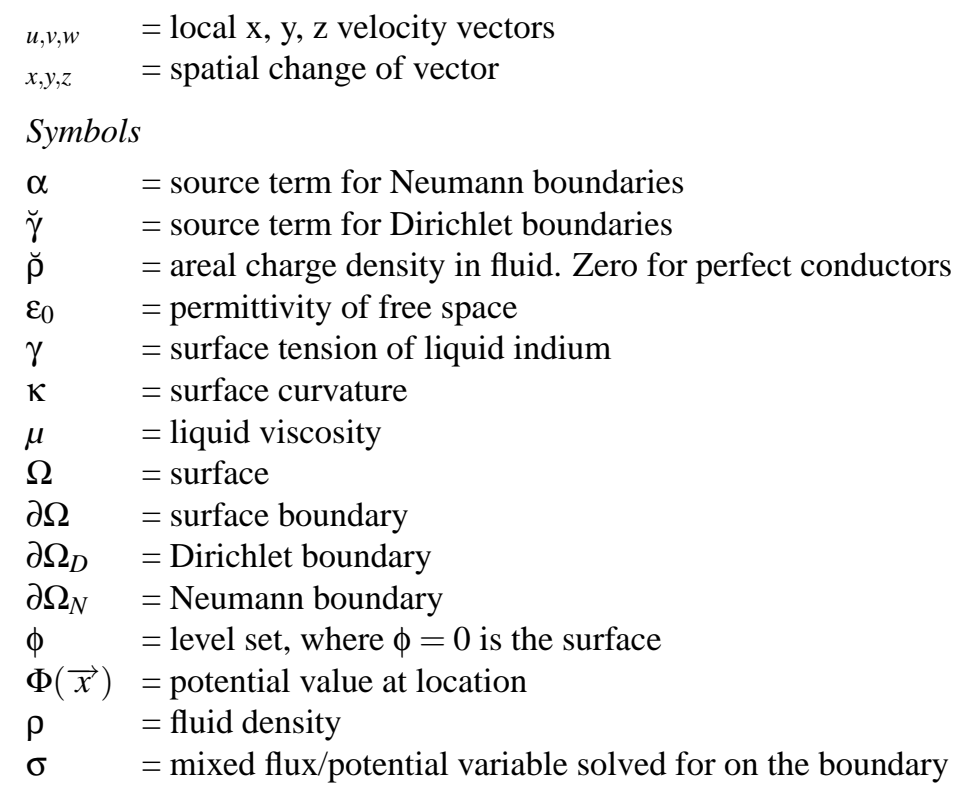

\section{Introduction}

Field emission electric propulsion (FEEP) thrusters are currently being considered for a variety of space missions both in the United States and Europe. They offer very low thrust levels $(\mu \mathrm{N})$, thrust repeatability $(<10 \mathrm{nN})$ and high accuracy impulse bits ( $\mathrm{nN}-\mathrm{s})$ combined with a very high mass efficiency $(8,000$ seconds specific impulse). Such thrusters are required for scientific drag-free missions such as LISA, ${ }^{1}$ Darwin, $\mathrm{GOCE}^{2}$ and SMART-2. A space-tested indium FEEP has been under development for over a decade. ${ }^{3}$

In a field emitter, the surface of a conductive liquid is exposed to a strong electrostatic field generating polarization forces that move the liquid in the field direction. The ensuing increase in surface area is counteracted by the surface tension of the fluid, forming an apex. ${ }^{4}$ When the field strength is on the order of $1 \frac{\mathrm{V}}{\mathrm{n}}$, surface ions are field evaporated and rapidly accelerated past the electrodes to final velocities of upwards of 50 kilometers per second, producing thrust in the vacuum of space. A ring electrode with a $-6 \mathrm{kV}$ potential around a fine tungsten needle about $1 \mathrm{~cm}$ long and $1 \mathrm{~mm}$ wide accelerates the liquid metal indium, as represented in Fig. (1). Experimental efforts indicate that below $20 \mu \mathrm{A}$ current, only ion emission occurs in FEEP needles. ${ }^{5}$ Above that point, at a current that varies based on the thermal and electrical properties of the fluid, periodic stochastic motions of droplet formation and emission interrupt the steady ion stream. The maximum current at which pure ion emission occurs is thus driven by the properties of the fluid flowing over the tip. As the current increases, ever-increasing mass fractions are emitted as large molecules and fewer as ions. Due to localized field evaporation on the needle shank, there is never $100 \%$ droplet content. For the emitter to be an effective attitude control thruster, a current of several hundred $\mu A$ is necessary. At currents this large, fluid instabilities occur and micro-droplets are emitted in addition to the ion current. ${ }^{6,7}$ Recent theory states that droplets begin to form when the velocity of indium being supplied to the tip is insufficient to replenish the mass lost through ion emission. ${ }^{8}$

The existence and corresponding behavior of droplets is of large practical concern because as more droplets form, operational efficiency decreases, lifetime is limited because drops clog the extractor electrode and plume divergence is impacted due to non-identical charge distributions in the exhaust particles. Therefore, a numerical investigation into the formation and charge distributions among these expelled droplets is being undertaken.

Simulating droplet snap-off is difficult because the region around the tip possesses characteristics that cause most numerical methods to fail. Examples of these characteristics are the presence of very large dynamic electrostatic gradients, rapid temporal change, highly non-symmetric geometry and infinite fluid curvature. To partially address these issues, front-tracking can be used (see Unverdi ${ }^{9}$ and Tryggvason ${ }^{10}$ ), but to sidestep them in the present work, a method involving interface tracking using level set computation is used that relies on the determination and movement of the boundary interface alone. For FEEP operation, those boundaries are located where the indium propellant is between a tungsten solid needle and a hard vacuum. The dominating forces on the propellant are surface tension, shear stress through viscosity and electrostatics. ${ }^{11}$ Both vacuum-indium and indium-tungsten surfaces can be followed with the level set method. ${ }^{12}$ The mathematical boundary integral method discretizes only the interface and is therefore 


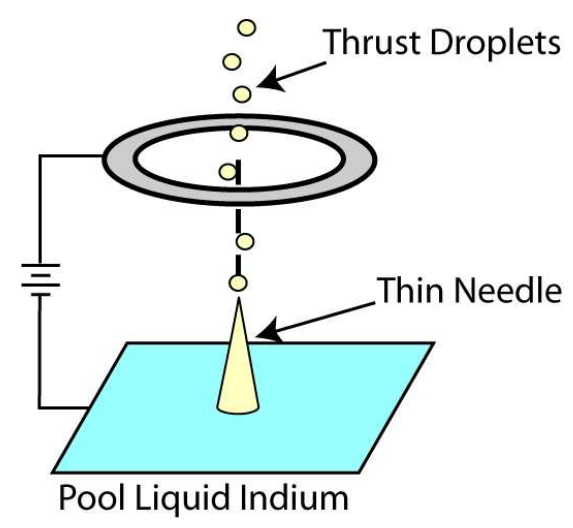

Figure 1. FEEP in droplet production mode

very efficient at producing a rapid and exact calculation of the normal electric field. However, if used alone, integral methods are limited in how they model viscosity and require complicated surface recomputing every time a boundary merges or splits. ${ }^{13}$

A combination of the level set and boundary integral methods provides a system that rapidly and accurately computes the electrostatically-induced movement of an interface without surface surgery.

\section{Level set model}

The level set model considers an incompressible, isothermal, viscous liquid. Indium is treated as a perfect conductor and its atoms are accelerated with ring electrodes producing thrust. The level set two dimensional governing equations for a FEEP are listed as Eqs. (1-4). The electric field is a surface normal force only, since $\vec{E}$ is zero inside a conductor. Mass and momentum are conserved with Eq. (1) and the free surface is advected with the pressure boundary condition, Eq. (4).

$$
\mathbf{U}_{t}+\mathbf{F}_{x}+\mathbf{G}_{y}=-\nabla p+q \overrightarrow{E_{n}}
$$

where the main variables are:

$$
\begin{gathered}
U=\left[\begin{array}{c}
\rho \\
\rho u \\
\rho v
\end{array}\right] \\
\mathbf{F}=\left[\begin{array}{c}
\rho u \\
\rho u^{2} \\
\rho u v
\end{array}\right], \mathbf{G}=\left[\begin{array}{c}
\rho v \\
\rho u v \\
\rho v^{2}
\end{array}\right] \\
p=\gamma \kappa+2 \mu_{\text {liquid }}\left(D_{\text {liquid }} \cdot \vec{n}\right) \cdot \vec{n}
\end{gathered}
$$

The subfunction variables $u, v, w$ are local velocity vectors along the $\mathrm{x}, \mathrm{y}$ and $\mathrm{z}$ axes, respectively. Surface tension $(\gamma)$ and viscosity $\left(\mu_{\text {liquid }}\right)$ are functions of the liquid temperature. Interface curvature is $\kappa^{14}$ and $D_{\text {liquid }}$ is the rate of deformation tensor for the liquid. Combining Eq. (1) with the general level set Eq. (5) allows the motion to be analyzed by convecting the $\phi$ levels (values) with the velocity field $\vec{v}^{15}$

$$
\phi_{t}+\vec{v} \cdot \nabla \phi=0
$$

where $\vec{v}$ is the desired velocity on the interface and is arbitrary elsewhere. Here, $\phi$ is the level set function which is positive in the liquid and negative in the vacuum. The equation (5) states that $\phi$ remains constant along particle paths, so $\phi=0$ always corresponds to the interface. The curvature of the interface $\kappa$ can then be described using the level set variable $\phi$ itself as

$$
\kappa=\left(\phi_{x x} \phi_{y}^{2}+\phi_{y y} \phi_{x}^{2}-2 \phi_{x} \phi_{y} \phi_{x y}\right) /\left(2\left(\phi_{x}^{2}+\phi_{y}^{2}\right)^{1.5}\right)
$$


The free surface is represented through a "coupled level set and volume-of-fluid" (CLSVOF) method. In addition to solving the level set equation (5), the volume-of-fluid function F is computed, ${ }^{15}$ using

$$
F_{t}+\mathbf{U} \cdot \nabla F=0
$$

where the net volume of fluid is conserved. $H$ is the Heaviside function evaluated at grid cells $i, j$ in Eq. (8).

$$
F_{i j}=\frac{1}{\Delta x \Delta y} \int_{\Omega_{i j}} H(\phi(x, y)) \partial x \partial y
$$

This work builds on prior three dimensional examples of level sets interface migration by Sussman, as illustrated in Fig. (2). These simulations do not include any electrostatic forces.
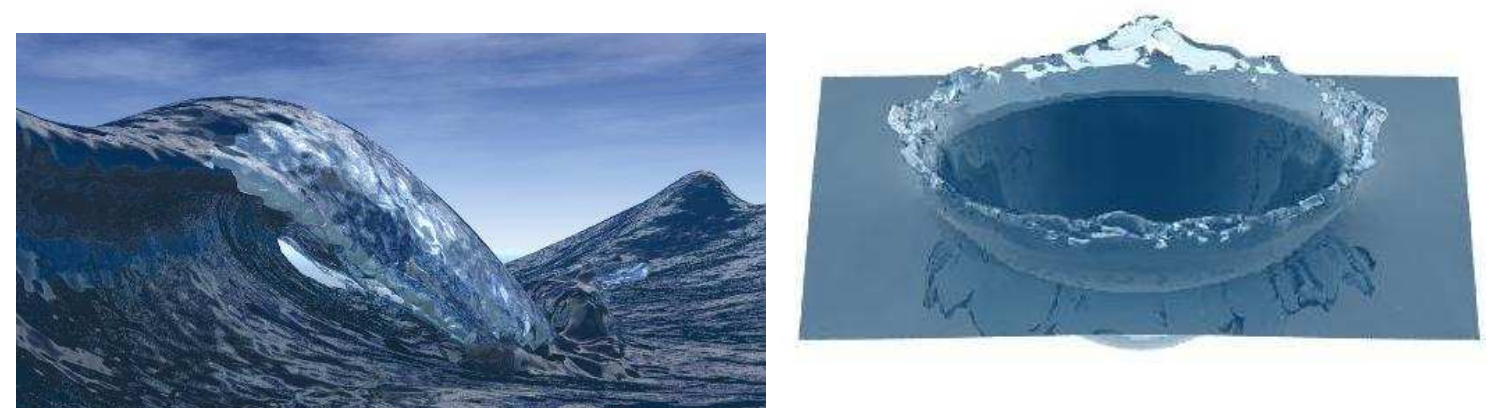

Figure 2. Sussman's wave level set simulation ${ }^{16}$

The efficient and exact calculation of the normal electric field $\overrightarrow{E_{n}}$ in Eq. (1) presents many challenges. A proposed algorithm for determining this electrostatic force for $N$ panels in $\mathrm{O}(N \ln N)$ time is the boundary integral method (BIM).

\section{Boundary integral method}

The boundary integral method rapidly and directly solves for the force on the surface without meshing the volume of the domain. It therefore avoids numerically noisy differentiation and can handle arbitrary geometries. For example, consider Poisson's equation

$$
\triangle \Phi(\bar{x})=\frac{\breve{\rho}}{\varepsilon_{0}},\left.\Phi\right|_{\partial \Omega}=\left.f \operatorname{or} \nabla \Phi\right|_{\partial \Omega} \cdot \vec{n}=g, \bar{x} \in \Omega
$$

where $\Phi$ is the electrostatic potential, $\breve{\rho}$ is the charge density, $\Omega$ the domain and $\partial \Omega$ the boundary.

\section{A. Green's function}

Let $\mathrm{G}\left(\bar{x} \mid \bar{x}_{0}\right)$ be the free space Green's function for the Laplace operator, which is the solution

$$
\triangle G=\delta\left(\bar{x}-\bar{x}_{0}\right)
$$

where $\bar{x}_{0}$ is a point on the interior of the domain and $\bar{x}$ is a boundary point. In two dimensional coordinates, the function becomes:

$$
G=-\frac{1}{4 \pi} \ln \left\{\left(x_{0}-x\right)^{2}+\left(y_{0}-y\right)^{2}\right\}
$$

Using Green's $2^{\text {nd }}$ identity:

$$
\iint_{A}(U \triangle V-V \triangle U) d A=\oint(U \nabla V-V \nabla U) \cdot \vec{n} d S
$$

and replacing $\mathrm{U}$ by the Greens function $(\mathrm{G})$ and $\mathrm{V}$ by $\Phi$ gives 


$$
\iint_{A} G \frac{\breve{\rho}(\bar{x})}{y} d A-\Phi\left(\bar{x}_{0}\right)=\oint\left(G\left(\partial_{\vec{n}} \Phi\right)-\Phi\left(\partial_{\vec{n}} G\right)\right) \cdot \vec{n} d S
$$

where $\partial_{\vec{n}} x=\nabla x \cdot \vec{n}$. Since we do not have any internal charge, $\breve{\rho}\left(\bar{x}_{0}\right)=0$ and the potential at any point can be calculated from the boundary flux and potential conditions. Carefully taking the limits of the single and double layered potential $^{17}$ gives the boundary integral formulation for LaPlace's equation from the inside of the domain.

$$
\frac{\Phi\left(\bar{x}_{0}\right)}{2}=\oint_{\partial \Omega} \Phi(\bar{x}) \partial_{n} G\left(\bar{x} \mid \bar{x}_{0}\right) \mathrm{d} s-\oint_{\partial \Omega} \partial_{n} \Phi(\bar{x}) G\left(\bar{x} \mid \bar{x}_{0}\right) \mathrm{d} s
$$

For most problems, $\partial \Omega$ will consist of a mixture of fixed potential (Dirichlet, $\partial \Omega_{D}$ ) and fixed flux (Neumann, $\partial \Omega_{N}$ ) conditions.

\section{B. Numerical discretization of the problem}

Approximating the integrals as a sum of panels, $\oint_{\partial \Omega_{D}} \Phi(\bar{x}) \partial_{n} G\left(\bar{x} \mid \bar{x}_{0}\right) \mathrm{d} s \approx \sum_{i=1}^{N_{D}} \Phi\left(\bar{x}_{i}\right) \oint_{\partial \Omega_{i}} \partial_{n} G\left(\bar{x} \mid \bar{x}_{0}\right) \mathrm{d} s$. If $\bar{x}_{1}$ is on a Dirichlet portion of the boundary, then we have

$$
\sum_{i=1}^{N_{D}} \partial_{n} \Phi\left(\overline{x_{i}}\right) \oint_{\partial \Omega_{i}} G\left(\bar{x} \mid \bar{x}_{1}\right) \mathrm{d} s-\sum_{i=1}^{N_{N}} \Phi\left(\bar{x}_{i}\right) \oint_{\partial \Omega_{i}} \partial_{n} G\left(\bar{x} \mid \bar{x}_{1}\right) \mathrm{d} s=\breve{\gamma}\left(\bar{x}_{1}\right)
$$

where $\breve{\gamma}\left(\bar{x}_{1}\right)$ are the known values given by

$$
\breve{\gamma}\left(\bar{x}_{1}\right)=-\frac{\Phi\left(\bar{x}_{1}\right)}{2}+\sum_{i=1}^{N_{D}} \Phi\left(\bar{x}_{i}\right) \oint_{\partial \Omega_{i}} \partial_{n} G\left(\bar{x} \mid \bar{x}_{1}\right) \mathrm{d} s-\sum_{i=1}^{N_{N}} \partial_{n} \Phi\left(\bar{x}_{i}\right) \oint_{\partial \Omega_{i}} G\left(\bar{x} \mid \bar{x}_{1}\right) \mathrm{d} s
$$

For $\bar{x}_{2}$ on a Neumann boundary, we have

$$
\frac{\Phi\left(\bar{x}_{2}\right)}{2}-\sum_{i=1}^{N_{N}} \Phi\left(\overline{x_{i}}\right) \oint_{\partial \Omega_{i}} \partial_{n} G\left(\bar{x} \mid \bar{x}_{2}\right) \mathrm{d} s+\sum_{i=1}^{N_{D}} \partial_{n} \Phi\left(\bar{x}_{i}\right) \oint_{\partial \Omega_{i}} G\left(\bar{x} \mid \bar{x}_{2}\right) \mathrm{d} s=\alpha\left(\bar{x}_{2}\right)
$$

where $\alpha\left(\bar{x}_{2}\right)$ are the known values given by

$$
\alpha\left(\bar{x}_{2}\right)=\sum_{i=1}^{N_{D}} \Phi\left(\overline{x_{i}}\right) \oint_{\partial \Omega_{i}} \partial_{n} G\left(\bar{x} \mid \bar{x}_{2}\right) \mathrm{d} s-\sum_{i=1}^{N_{N}} \partial_{n} \Phi\left(\overline{x_{i}}\right) \oint_{\partial \Omega_{i}} G\left(\bar{x} \mid \bar{x}_{2}\right) \mathrm{d} s
$$

The matrix to solve becomes

$$
\left[\begin{array}{cc}
a_{1,1} . . a_{1, N_{D}} & b_{1,1} . . b_{1, N_{N}} \\
\frac{a_{N_{D}, 1} . . a_{N_{D}, N_{D}}}{c_{1,1} . . c_{1, N_{D}}} & \frac{b_{N_{D}, 1} . . b_{N_{D}, N_{N}}}{d_{1,1} . . d_{1, N_{N}}} \\
c_{N_{N}, 1} . . c_{N_{N}, N_{D}} & d_{N_{N}, 1} . . d_{N_{N}, N_{N}}
\end{array}\right]\left[\begin{array}{c}
\partial_{n} \Phi\left(\bar{x}_{1,1}\right) \\
. . \\
\partial_{n} \Phi\left(\bar{x}_{1, N_{D}}\right) \\
\Phi\left(\bar{x}_{2,1}\right) \\
. . \\
\Phi\left(\bar{x}_{2, N_{N}}\right)
\end{array}\right]=\left[\begin{array}{c}
\breve{\gamma}\left(\bar{x}_{1,1}\right) \\
. . \\
\breve{\gamma}\left(\bar{x}_{1, N_{D}}\right) \\
\alpha\left(\bar{x}_{2,1}\right) \\
. . \\
\alpha\left(\bar{x}_{2, N_{N}}\right)
\end{array}\right]
$$

where the values in the matrix are given by Eq. (20).

$$
\begin{array}{lc}
a_{i, j}= & \int_{\partial \Omega_{D}} G\left(\bar{x}, \mid \bar{x}_{1, j}\right) \mathrm{d} s \\
b_{i, j}= & -\int_{\partial \Omega_{N}} \partial_{n} G\left(\bar{x}, \mid \bar{x}_{1, j}\right) \mathrm{d} s \\
c_{i, j}= & \int_{\partial \Omega_{D}} G\left(\bar{x}, \mid \bar{x}_{2, j}\right) \mathrm{d} s \\
d_{i, j}= & -\int_{\partial \Omega_{N}} \partial_{n} G\left(\bar{x}, \mid \bar{x}_{2, j}\right) \mathrm{d} s \\
\breve{\gamma}\left(\bar{x}_{1}\right)= & -\frac{\Phi\left(\bar{x}_{1}\right)}{2}+\oint_{\partial \Omega_{D}} \Phi\left(\overline{x_{i}}\right) \partial_{n} G\left(\bar{x} \mid \bar{x}_{1}\right) \mathrm{d} s-\oint_{\partial \Omega_{N}} \partial_{n} \Phi\left(\overline{x_{i}}\right) G\left(\bar{x} \mid \bar{x}_{1}\right) \mathrm{d} s \\
\alpha\left(\bar{x}_{2}\right)= & \oint_{\partial \Omega_{D}} \Phi\left(\bar{x}_{i}\right) \partial_{n} G\left(\bar{x} \mid \bar{x}_{2}\right) \mathrm{d} s-\oint_{\partial \Omega_{N}} \partial_{n} \Phi\left(\overline{x_{i}}\right) G\left(\bar{x} \mid \bar{x}_{2}\right) \mathrm{d} s
\end{array}
$$

The potential at any arbitrary point in the domain is thus given by summing the contributions from fixed potential (Dirichlet=D) and fixed flux (Neumann=N) boundaries, as in Eq. (14). 


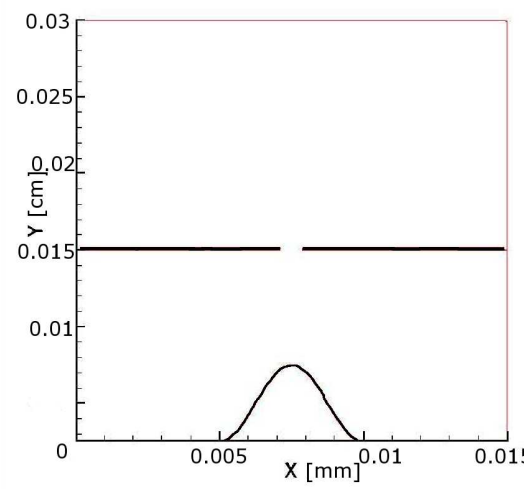

Figure 3. Computational panel location, in meters.

The computational domain paneled is shown in Fig. (3). With a level set grid of $256 \times 512$ cells, each cell is about $1-1.5 \times 10^{-5} \mathrm{~m}$. With 1900 panels, the average panel length is $3 \times 10^{-5} \mathrm{~m}$. The droplets described are all $1 \times 10^{-4} \mathrm{~m}$ diameter or larger, so both the cell size and panel length are significantly smaller than the droplets. Further resolution refinement does not change the results up to pinch off, but due to slight asymmetries in the automatic boundary meshing routine, there is a slight asymmetry left to right after pinch-off. A further refinement study is underway.

\section{Solving for the normal electric field directly}

One of the main advantages of the BIM is the direct interface force calculation as a direct result of the problem formulation. Solving the matrix Eq. (19) for the $\partial_{n} \Phi\left(\bar{x}_{1}\right)$ term directly for the normalized test shape in Fig. (4a) provides the normal component of the electric field $(\sigma)$ on the surface $x \in\{0,1\} \cup y \in[0.2,0.8]$, as seen in Fig. (4b).

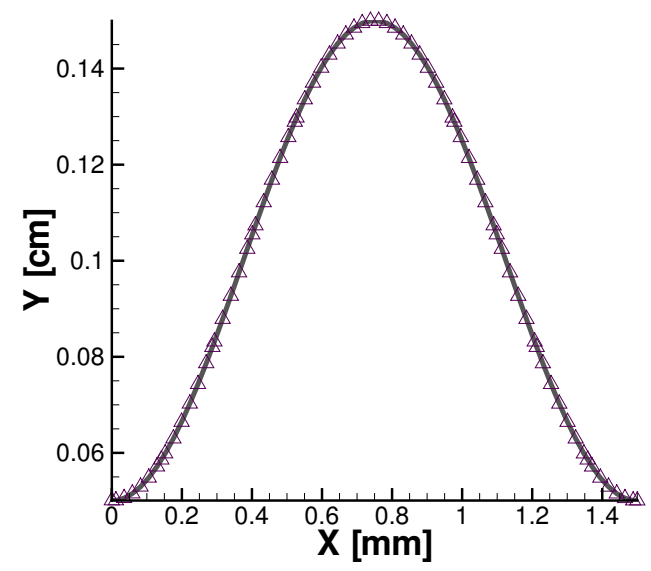

(a) Normalized test shape boundary nodes

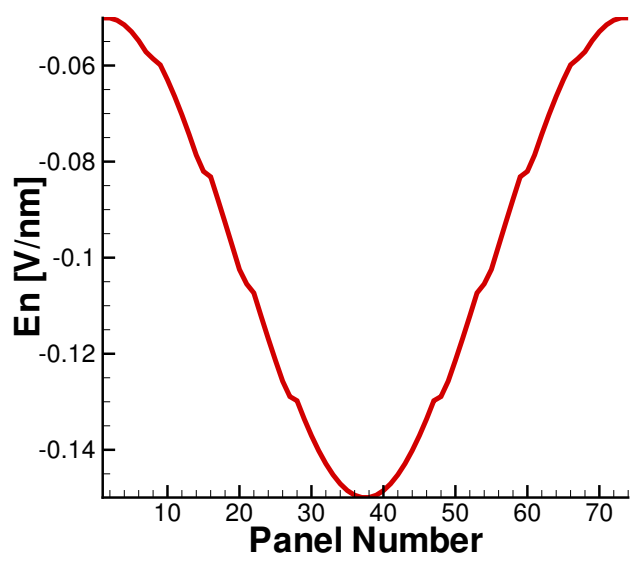

(b) Normal electric field for curved surface

Figure 4. Boundary nodes and normal electric field along curved surface of test shape

Gauss' Law says that the total flux of the electric field through an element is

$$
\Phi_{n e t}=\oint \vec{E} \cdot \vec{n} d A
$$

This field comes from the total interior charge $Q_{\text {inside }}$, so the net potential is $\Phi_{n e t}=\vec{E}_{n} L=\frac{Q_{\text {inside }}}{\varepsilon_{0}}$. Solving for the 
charge, $Q_{\text {inside }}=\varepsilon_{0} \vec{E}_{n} L$. Drawing a box around each panel, the assumption of the fluid being a perfect conductor means that all flux is out of the surface and there is not a tangential electric field. Therefore, the entire contribution of potential comes from the normal electric field times the panel length, L. Therefore, the total force experienced on the panel is given by:

$$
F=Q E=\varepsilon_{0} L \vec{E}_{n}^{2}
$$

This is the desired force we were originally looking for, with no introduced gradient noise and not requiring a surrounding grid. It holds for any complex geometry and curves.

\section{Results}

Using Eq. (22), the force on the surface can be calculated and then advected forward in time with Eqs. (1, 5, 7). Indium FEEP droplets are formed as a function of space and time, as seen in the following figures. The base computational case is a two dimensional field emitter with indium having a viscosity of $0.0017 \frac{\mathrm{N} \cdot \mathrm{s}}{\mathrm{m}^{2}}$, surface tension of $0.552 \frac{\mathrm{N}}{\mathrm{m}}$ and an electric field at the tip of $40 \frac{\mathrm{V}}{\mathrm{nm}}$. This is higher than the experimentally observed electric fields of $1 \frac{\mathrm{V}}{\mathrm{nm}}$, due to the simulation being a 2D planar slice instead of a ring electrode surrounding the emitting needle. The electrostatic force comes largely from 2 points at the edges of the electrode instead of equally surrounding the needle tip, resulting in unrealistic 2D force vectors. Figure (5) shows the fluid accelerating at about $4 \times 10^{13} \frac{\mathrm{m}}{2}$, and now traveling at $150 \frac{\mathrm{km}}{\mathrm{s}}$. The rectangle at the top of Fig. (5a) represents the position of the electrodes and the triangle in the bottom center is the underlying solid tungsten needle.

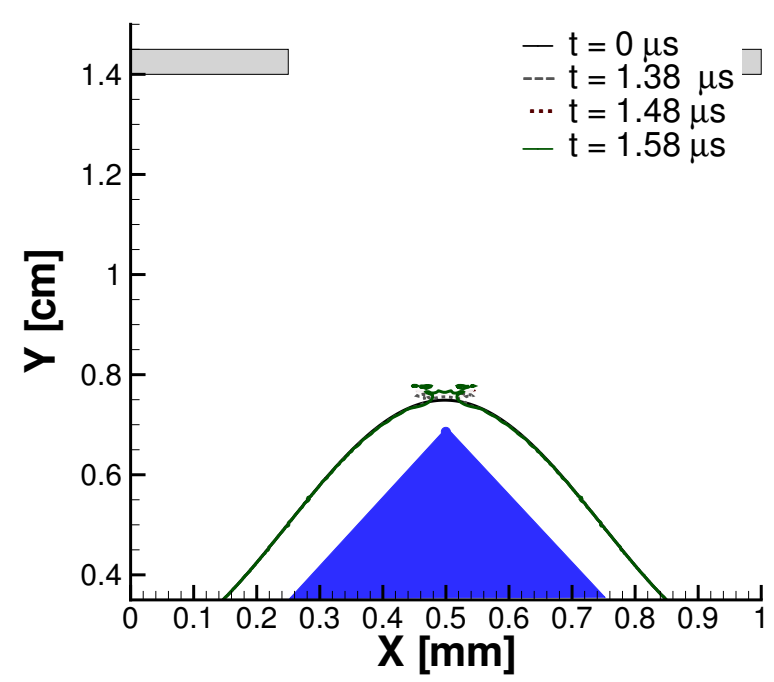

(a) Full picture of droplet formation

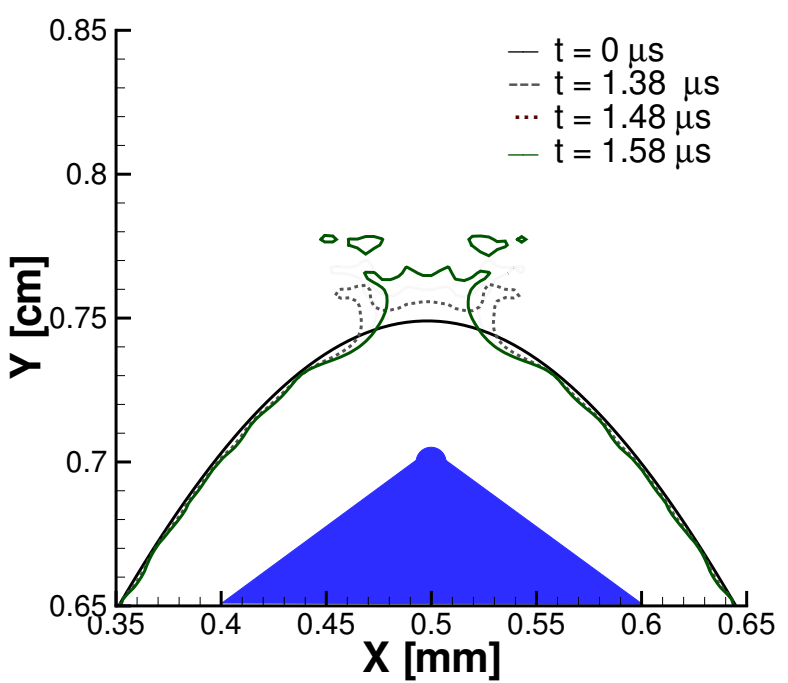

(b) Zoomed in case of droplet acceleration

Figure 5. Droplet formation and acceleration, base case

Figure (6) displays the instantaneous velocity vector fields at a) $3.12 \mu \mathrm{s}$ and b) $3.39 \mu \mathrm{s}$. Note the higher cusp and droplets detaching and moving away from the needle tip as compared to the earlier time vector field.

\section{A. Viscosity}

Indium's ability to flow over a surface is determined largely by its viscosity; it is sensitive to temperature as follows:

$$
\mu_{\text {In }}\left[\frac{N \cdot s}{m^{2}}\right]=3 \times 10^{-4} e^{800 / T[K]}
$$




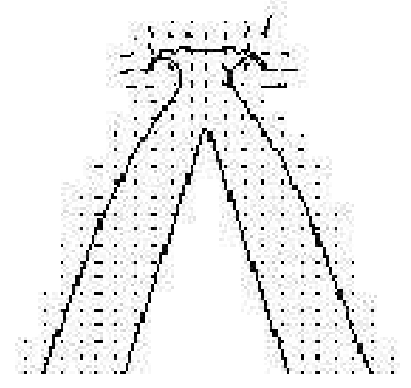

(a) Velocity at time $=3.12 \mu \mathrm{s}$

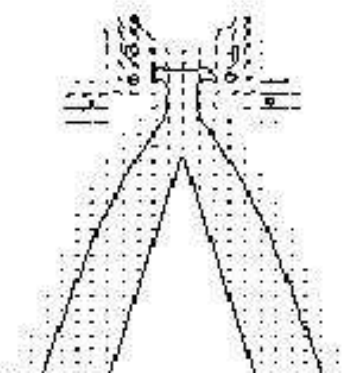

(b) Velocity at time $=3.39 \mu \mathrm{s}$

Figure 6. Velocity field on droplets

The viscosity of the simulations is varied by an order of magnitude lower and higher than the baseline behavior and the results are shown in Fig. (7). The lower viscosity occurs around 2,000 K while the higher value exists at $200 \mathrm{~K}$. As the fluid becomes thinner at higher temperatures, it forms into droplets sooner in time and space, and the disturbance to the fluid moves down the needle much sooner.

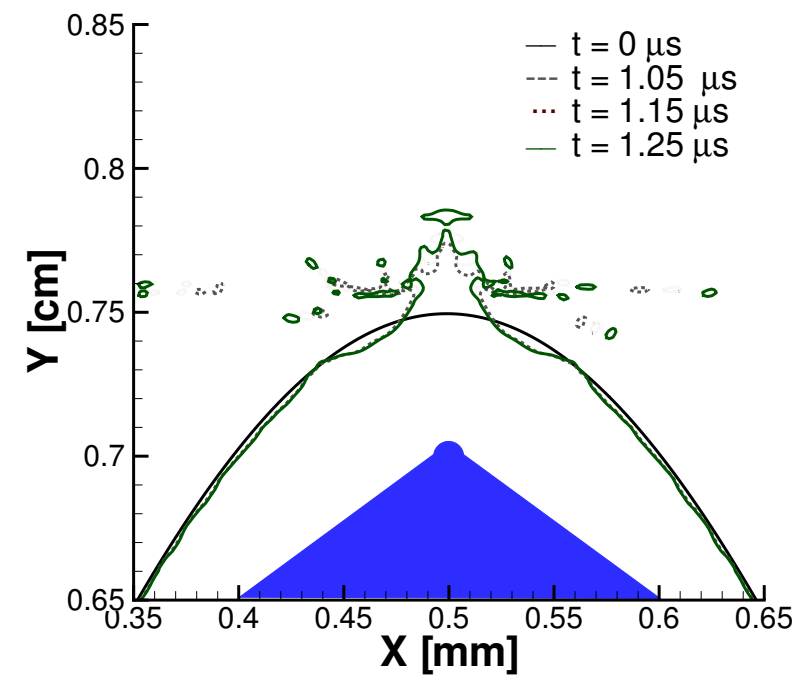

(a) Low viscosity, $\mu=0.00017 \frac{\mathrm{N} \cdot \mathrm{S}}{\mathrm{m}^{2}}$

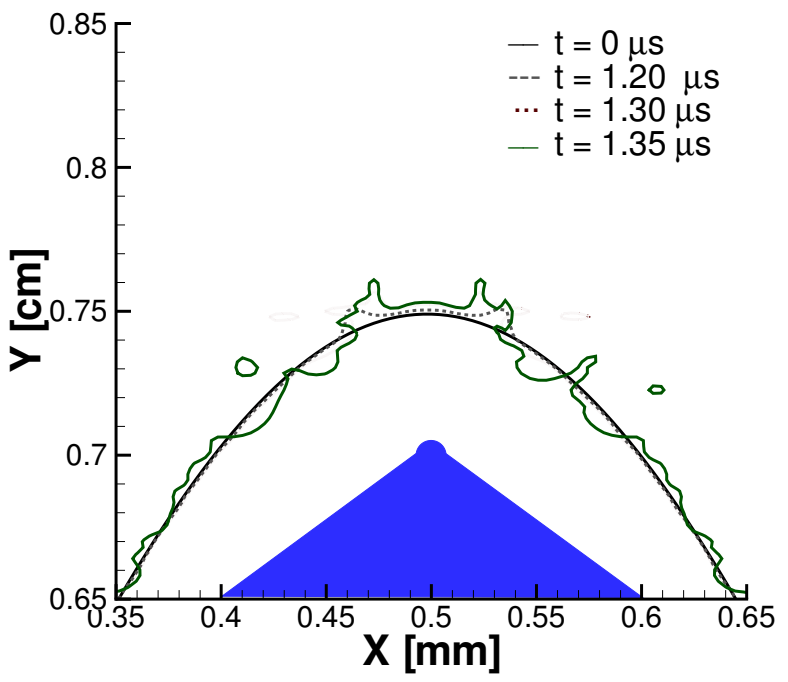

(b) High viscosity, $\mu=0.017 \frac{\mathrm{N} \cdot \mathrm{s}}{\mathrm{m}^{2}}$

Figure 7. Effect of viscosity on droplet evolution

\section{B. Surface tension}

Surface tension of the fluid does not vary rapidly with temperature at all, as indicated by:

$$
\gamma_{I n}\left[\frac{N}{m}\right]=\frac{555-0.12(T[K]-430)}{1000}
$$

If the temperature were to increase by $1,500 \mathrm{~K}$ to the $2,000 \mathrm{~K}$ fluid temperature seen in the low viscosity case, surface tension would drop by only one-third for $\gamma_{I n}$. To determine the gross effect of this parameter, simulations are 
performed in which the surface tension is varied by an order of magnitude lower and higher and the results are shown in Fig. (8). At very high values of $\gamma_{I n}$, the droplets snap off much faster, as the high cohesion and tension of the surface interface strongly pulls the evolving surface into droplets, minimizing the internal energy. However, the path of development is similar to the base calculation, Fig. (5).

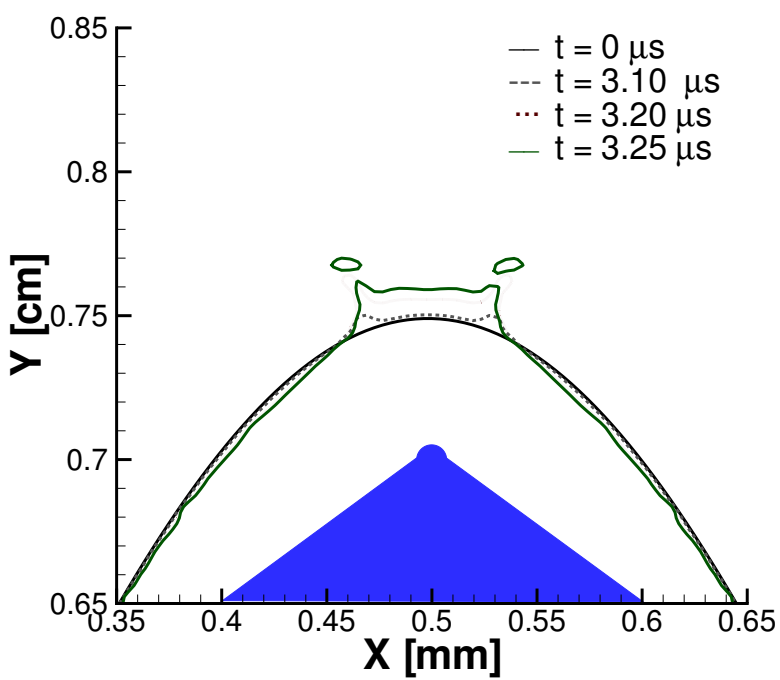

(a) Low surface tension, $\gamma=0.055 \frac{\mathrm{N}}{\mathrm{m}}$

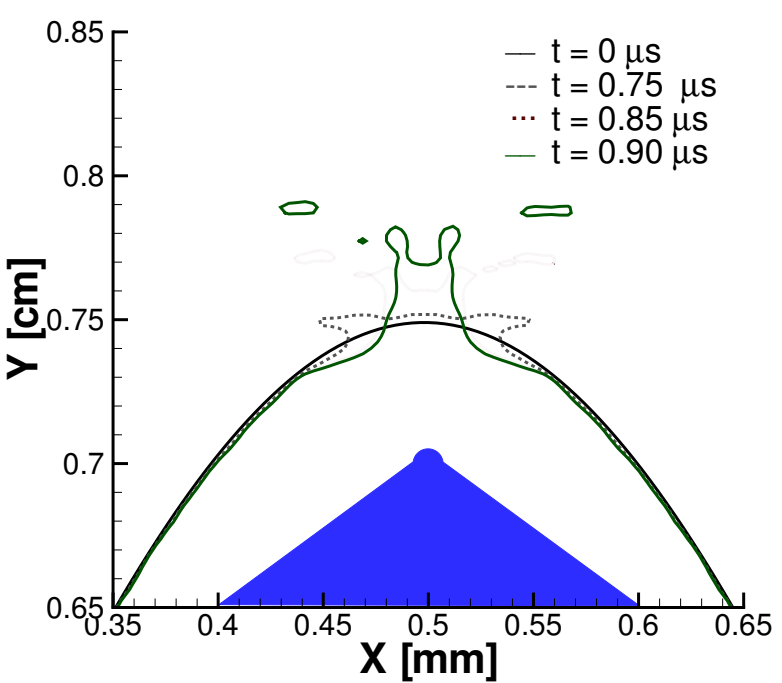

(b) High surface tension, $\gamma=5.5 \frac{\mathrm{N}}{\mathrm{m}}$

Figure 8. Effect of surface tension on droplet evolution

\section{Size of axial gap}

The width of the gap between the axis centerline and the edge of the electrode has a smaller impact on the shape of the droplet pulled off the tip. As the gap approaches zero and the electrodes resemble a flat plate, the corresponding electric field is almost parallel and a pronounced anvil head forms. As the electrode widens, the droplet is pulled towards the edges preferentially, both at the surface itself and after droplet separation. The axial gap between simulations is varied by over an order of magnitude and the results are displayed in Fig. (9).

\section{Electric field}

As expected, the strength of the normal electric field strongly influences the speed of formation, behavior and shape of indium droplets. Figure (10a) displays droplet evolution at $\frac{1}{100}$ th the base electrode potential; the simulated time before droplet detachment is ten times greater than in Fig. (5). The droplets accelerate and are now moving at 290 $\frac{m}{s}$. Note the thin stream going directly vertical as the relatively slow pull allows a local surface equilibrium. At an electrode potential $\frac{1}{10}$ th the base electrode potential, Fig. (10b) displays droplets accelerating and are now traveling at $15 \frac{\mathrm{km}}{\mathrm{s}}$. There is a strong two-stream pull towards the edge of the two electrodes in this simulation.

\section{Conclusion}

Tying together the physical FEEP geometry of Fig. (1) with an existing level set code ${ }^{15}$ allowed for the simulation of liquid indium forming a droplet off a field emitter tip. The boundary integral method provided a rapid calculation of the external potentials and electric fields, thereby driving the propellant's evolution. The combination of the two methods provided accurate simulation of a two dimensional electrode forming droplets off the needle tip. The qualitative effects of varying viscosity, surface tension, size of axial gap and electrode potential were investigated. 


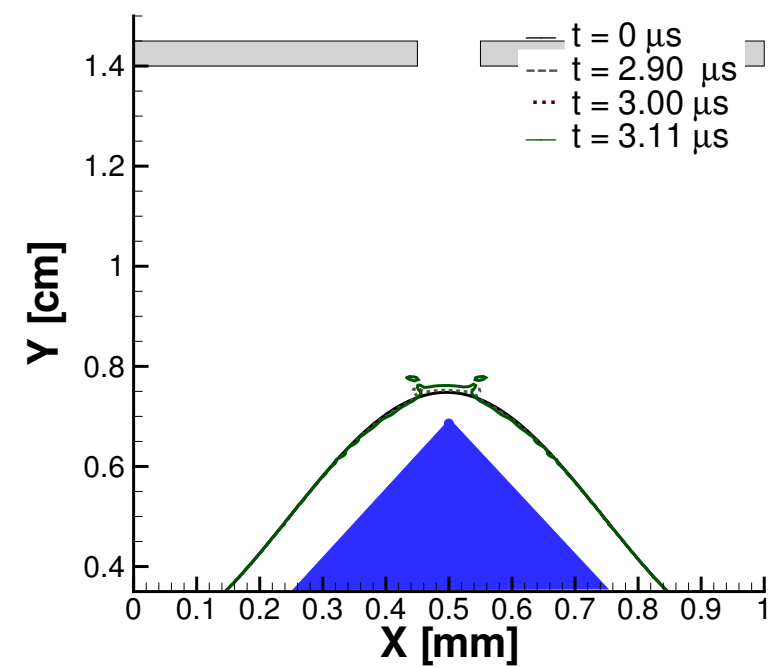

(a) Small axial gap, $\delta=0.1 \mathrm{~mm}$

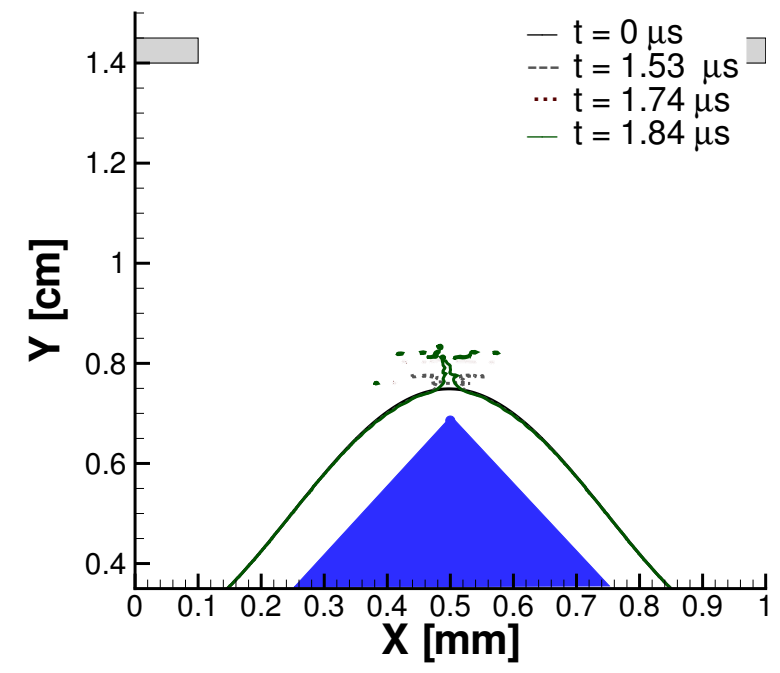

(b) Large axial gap, $\delta=0.8 \mathrm{~mm}$

Figure 9. Effect of size of axial gap on droplet evolution

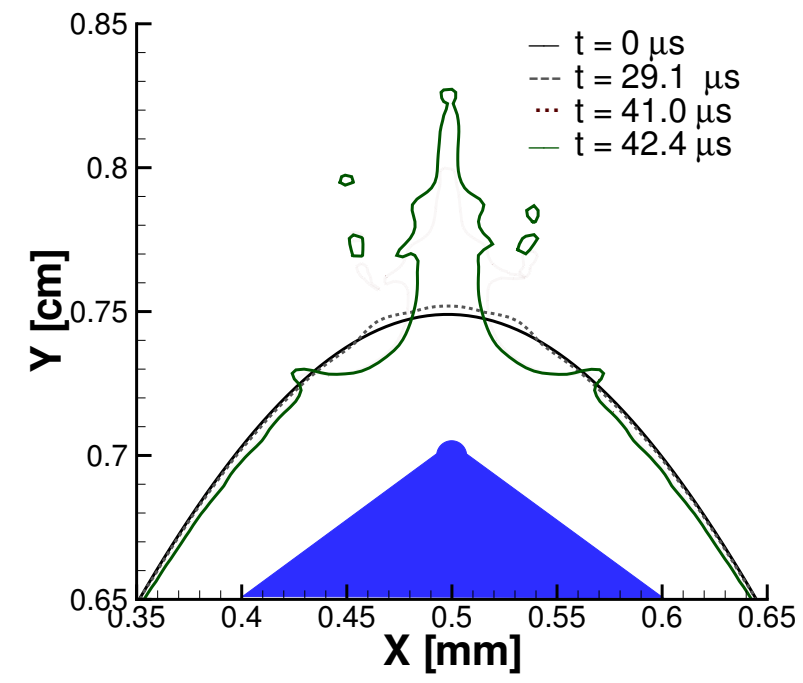

(a) Low electric field, $\mathrm{E}=0.4 \mathrm{~V} / \mathrm{nm}$

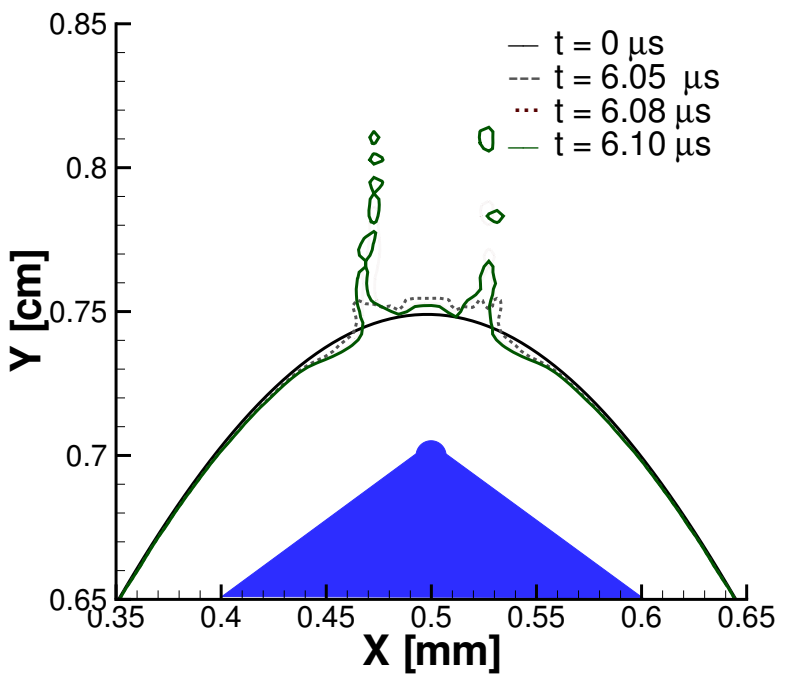

(b) Medium electric field, $\mathrm{E}=4.0 \mathrm{~V} / \mathrm{nm}$

Figure 10. Effect of electric field on droplet evolution

10 of 11 
In on-going work, the 2D planar droplet formulation is being expanded into a full 3D non axisymmetric description where droplet sizes and charges will be simulated quantitatively. The panel interactions in Eq. (20) are being replaced with a tree code ${ }^{18}$ so that future calculations are performed without a connection matrix being necessary. The spacings on the level set being passed back and forth are going to be uniform. Therefore, a shape with arbitrary geometry and apriori unknown panel connections can be advected forward in time accurately including gravity and electrostatic forces without requiring surface reconstruction or time step intervention.

\section{Acknowledgments}

The first author thanks the NASA Jet Propulsion Laboratory for funding through the NASA Graduate Student Research Program.

\section{References}

${ }^{1}$ Berti, E., Buonanno, A., and Will, C., "Estimating spinning binary parameters and testing alternative theories of gravity with LISA," Physical Review D, Vol. 71, No. 8, April 2005.

${ }^{2}$ Kern, M. and et. al., "Outlier detection algorithms and their performance in GOCE gravity field processing," J. Geodesy, Vol. 78, No. 9, April 2005, pp. 509-519.

${ }^{3}$ Genovese, A., Tajmar, M., and Steiger, W., "Indium FEEP Endurance Test: Preliminary Results," No. IEPC-01-289 in IEPC 27th International Electric Propulsion Conference, Pasadena, CA, October 2001.

${ }^{4}$ Rudenauer, F., Fehringer, M., and Steiger, W., "Indium field emission microthrusters," No. SP-398 in Proceedings of the Second European Spacecraft Propulsion Conference, 27 May 1997, pp. 267-271.

${ }^{5}$ Genovese, A., Steiger, W., and Tajmar, M., "Indium FEEP Microthruster: Experimental Characterization in the 1-100 $\mu$ N Range," Vol. AIAA 2001-3788 of 37th Joint Propulsion Conference, 8-11 July 2001, pp. 1-12.

${ }^{6}$ Tajmar, M. and Genovese, A., "Experimental evaluation of a mass-efficiency model for an indium liquid-metal ion source," Journal Physics A, Vol. 76, 2003, pp. 1003-1006.

${ }^{7}$ Vladimirov, V. and Gorshkov, V., "Stability of conducting beams in ionic sources on liquid metals," Journal of Technical Physics, Vol. 57, No. 11,1987 , pp. 2155-2163.

${ }^{8}$ Lopey-Herrera, J., Ganan-Calvo, A., and Perez-Saborid, M., "One-dimensional simulation of the breakup of capillary jets of conducting liquids. Application to EHD spraying," Journal Aerosol Science, Vol. 30, No. 7, 1999, pp. 895-912.

${ }^{9}$ Unverdi, S. and Tryggvason, G., "A front-tracking method for viscous, incompressible, multi-fluid Flows," J. Computational Physics, Vol. 100, 1992, pp. 25-37.

${ }^{10}$ Tryggvasonm, G., Bunner, B., Juric, D., and Tauber, W., "A front tracking method for computations of multiphase flows," J. Computational Physics, Vol. 169, 2001, pp. 708-759.

${ }^{11}$ VanderWyst, A., "Perturbation model of slender jets," Asyntotic analysis of FEEP governing forces.

${ }^{12}$ Sussman, M., Smereka, P., and Osher, S., "A level set approach for computing solutions to incompressible two-phase flow," J. Computational Physics, Vol. 114, 1994, pp. 146-159.

${ }^{13}$ Best, J., "The formation of toroidal bubbles upon the collapse of transient cavities," J. Fluid Mechanics, Vol. 251, 1993, pp. 79-107.

${ }^{14}$ Farag, A. and Hassan, H., “Adaptive Segmentation of Multi-model 3D Data Using Robust Level Set Techniques," MICCAI LNCS, Vol. 3216 , 2004, pp. 143-150.

${ }^{15}$ Sussman, M., "A second order coupled level set and volume-of-fluid method for computing growth and collapse of vapor bubbles," $J$. Computational Physics, Vol. 187, 2003, pp. 110-136.

${ }^{16}$ Symposium on computer animation (SCA), Animation and control of breaking waves, ACM SIGGRAPH/Eurographics, Paris, France, 2004.

${ }^{17}$ Folland, G., Introduction to Partial Differential Equations, Princeton University Press, 1979.

${ }^{18}$ Christlieb, A., Krasny, R., and Verboncoeur, J., "A treecode algorithm for simulating electron dynamics in a Penning-Malmberg trap," Computer Physics Communications, Vol. 164, No. 1-3, 1 Dec. 2004, pp. 306-310. 